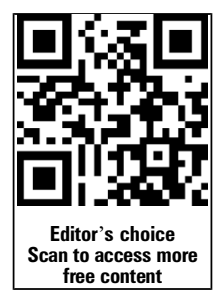
free content
- Additional material is published online only. To view please visit the journal online (http://dx.doi.org/10.1136/ bjsports-2015-095073).

${ }^{1}$ Aspetar Sports Medicine Hospital, Doha, Qatar

${ }^{2}$ Department of Rehabilitation Science and Physiotherapy,

Faculty of Medicine and Health Science, Ghent University, Ghent, Belgium

${ }^{3}$ Amsterdam Center of Evidence Based Sports Medicine, Academic Medical Center, Amsterdam,

The Netherlands

\section{Correspondence to} Dr Rod Whiteley, Aspetar Sports Medicine Hospital, P.O. Box 29222 Doha 29222, Qatar;

rodney.whiteley@aspetar.com

Accepted 11 January 2016 Published Online First

3 February 2016

\title{
A combination of initial and follow-up physiotherapist examination predicts physician-determined time to return to play after hamstring injury, with no added value of MRI
}

\author{
Phil Jacobsen, ${ }^{1}$ Erik Witvrouw, ${ }^{1,2}$ Patrice Muxart, ${ }^{1}$ Johannes L Tol, ${ }^{1,3}$ Rod Whiteley ${ }^{1}$
}

\begin{abstract}
Background Previous studies investigating prediction of return to play after acute hamstring injury were limited by examining a single postinjury clinical and MRI evaluation. We evaluated the added value of including follow-up clinical evaluation when predicting return to play.

Methods A range of clinical and MRI parameters were prospectively investigated for an association with the time to return to play in 90 athletes with MRI positive hamstring injuries undergoing a criteria-based rehabilitation programme. Clinical evaluation was performed within 5 days of injury and 7 days after this initial assessment (follow-up clinical evaluation). The association between possible prognostic parameters and the time to return to play was assessed with a multiple linear regression model.
\end{abstract}

Results Data of 90 athletes were available for analysis. At the first physiotherapy appointment, a combination of three demographic and six clinical variables explained $50 \%$ of the variance ( \pm 19 days) in the time to return to play. At follow-up assessment (7 days), a combination of 10 clinical and demographic variables explained $97.0 \%$ of the variance ( \pm 5 days) in time to return to play. In order of importance, the variables were: change in strength during the first week for the 'mid-range' test, peak isokinetic knee flexion torque of the uninjured leg, maximum pain at the time of injury, number of days to walk pain free, playing the sport of football, strength performing the 'inner range' hamstring test at day 1 , presence of pain on a single leg bridge at day 7 or its absence during a single leg bridge, delay in starting treatment and percentage of strength in the 'outer range' test compared to the healthy leg. No MRI variables were retained in any of these analyses. MRI variables alone explained $8.6 \%$ of the variance-which is unhelpful to players and coaches.

Summary The combination of initial and 7-day followup clinical evaluation is clinically helpful in predicting time to return to play ( \pm 5 days) following acute hamstring injury. MRI offered no useful clinical information regarding return to play duration in this cohort.

Trial registration number NCT01812564.

\section{INTRODUCTION}

Return to play after hamstring strain injury varies widely_from 1 week $^{1}$ to over 50 weeks $^{2}$ or longer ${ }^{3}$ — despite similar clinical grading and sporting requirements. Such variability is a significant challenge in the professional sporting environment -the players and their coaches, managers and others feel the clinical team should be able to estimate the likely time loss from the injury.

A number of factors have been proposed as influencing the time loss after hamstring injury, including: the mechanism of injury, ${ }^{4}$ injury location, ${ }^{5}$ MRI grading of injury, ${ }^{6}$ as well as the involvement of the proximal tendon.

Whether variations in rehabilitation protocols will influence the duration of RTS is not known. ${ }^{8-}$ ${ }^{10}$ Data from Askling et $a l^{9}{ }^{10}$ suggest that adding early outer-range eccentric exercise to usual care shortens rehabilitation time and lowers reinjury rates, but this research did not control for the other exercises performed by the injured athletes. Sherry and $\mathrm{Best}^{8}$ tested an intervention but their study had a small sample sizes so a difference in return to play of 20 days was statistically insignificant.

Our goal was to examine the ability of (1) subjective and objective information obtained at the time of initial physiotherapy examination, (2) results of physiotherapy examination 7 days after the initial examination and (3) the MRI examination at initial examination to predict time to return to play after hamstring injury. A strength of our study design was that all participants underwent a standardised, criterion-based rehabilitation protocol with standard return to play criteria (these are detailed in the online web appendix). The present report is a preplanned secondary analysis of data acquired during the Aspetar PRP trial (Registered trial number: ClinicalTrials.gov Identifier: NCT01812564). ${ }^{11}$

\section{METHODS}

This is a substudy of a double-blind randomised controlled trial examining the additional effect of acute injection of platelet-rich plasma and standardised physiotherapy (PRP+Physio), compared to platelet-poor plasma and standardised physiotherapy (PPP + Physio) and standardised physiotherapy without injection (Physio) on the return to play time and reinjury after MRI confirmed grade 1 and 2 hamstring injury. ${ }^{11}$

Inclusion criteria were male athletes who: (1) presented to our outpatient physiotherapy service within 5 days with an MRI-confirmed acute hamstring injury, (2) agreed to be randomised to receive one of the three interventions and (3) consented to physiotherapy treatment 5 days per week. The full methodology for the randomised trial are detailed elsewhere. $^{11}$
Witvrouw $E_{1}$ Muxart $P$, et al. Br J Sports Med 2016:50:431-439. 
Table 1 Summary of the assessment items that were candidates for the regression analyses, and the results

\section{Assessment item}

Injury and patient history

Date of injury and today's date (to calculate delay in presentation)

Sport played

Side injured and limb dominance

Onset of pain-sudden or gradual

Pain local to hamstring only or referred

Injury incurred during game or training

Was the athlete forced to stop within $5 \mathrm{~min}$ ?

Was the athlete able to keep running after the injury?

Could the athlete walk pain free immediately?

How many days until the athlete was able to walk pain free?

Mechanism of injury (sprinting, kick, tackle, stretch or other)

Was the injured leg weight-bearing at the time of injury?

Maximum pain at the time of injury? (0-10)

Maximum pain today (day of assessment) (0-10)

Previous history of hamstring injury to this leg or the other leg? If yes, include duration of absence from sport.

History of low back pain?

History of knee injury on this leg or the other leg?

History of groin injury to this side or the other side?

History of calf injury to this side or the other side?

History of other injuries not specified above?

History of surgery?

Physical examination of current injury

How far can the athlete forward flex while standing (knee, mid shin, ankles, floor) and do they have pain limiting?

Can the athlete walk without pain and/or a limp?

Can the athlete jog without pain and/or a limp?

Is there any pain on a 2 leg half squat?

Is there any pain on a 1 leg quarter squat?

Is the pain on palpation of the injured area nil, mild, or marked?

What is the length (in $\mathrm{cm}$ ) of pain craniocaudally on palpation?

What is the width of pain (in $\mathrm{cm}$ ) medial-laterally on palpation?

What is the distance from the ischium (in $\mathrm{cm}$ ) of the most proximal aspect of the patient's pain?

What is the strength (in $\mathrm{kg}$ ) of both, the injured and uninjured leg on the 'inner', 'mid' and 'outer' range tests?

Is there pain on any of these strength tests, if so, on which?

What is the range of motion (in degrees) for: Straight Leg Raise, Passive Knee Extension in $90^{\circ}$ hip flexion and Active Knee Extension in maximum hip flexion?

Is there any pain on any of these tests, if so, on which?

Can the athlete perform 3 repetitions of a single leg bridge on a $1 \mathrm{~m}$ step painlessly? If not, how close can he get-3 repetitions on the bed with 1 leg, 3 repetitions with 2 legs, 2 legs partial movement, or only reaching the starting position?

Isokinetic evaluation of the uninjured leg recording peak torque and angle of peak torque for knee flexion and extension at $60 \% \mathrm{~s}, 300 \% \mathrm{~s}$ concentric, and $60 \% \mathrm{~s}$ eccentric (knee flexion only) MRI variables: volume of oedema, craniocaudal length of oedema, medial-lateral length, tendon involvement, myofascial involvement, muscle involved, per cent involvement compared to entire muscle, distance to ischium of most proximal part of injury

Retained in initial examination Retained in week 1 regression regression analysis?

analysis?

No

If volleyball
No
No
No
No
No
No
№
№
No
No
No
Yes

\section{If both legs had previously} had a hamstring injury

No

No

No

No

No

No

No

No

No

No

No

No

No

No

No

Mid range strength expressed as a percentage of the uninjured leg

Pain on outer range strength test at day 1

Straight leg raise;

Passive knee extension range on the uninjured leg

No

No

Angle of peak torque for knee extension at $60 \% \mathrm{~s}$

No
Yes (days delay)

If football

No

No

No

No

No

No

No

Yes

No

No

No

Yes

No

No

No

No

No

No

No

No

No

No

No

No

No

No

No

Change in strength for the mid range test in the first week; inner range strength on day 1 ; outer range strength as a percentage at day 7

If the outer range test is painless at day 7

No

No

If the athlete can do the single leg bridge painlessly at day 7

Peak torque for knee flexion at $60 \%$ $\mathrm{s}$, concentric

No
All participants were given a standardised, criteria-based rehabilitation protocol (see online supplementary material) under the supervision of three experienced sports physiotherapists. Treating physiotherapists were blinded to the MRI results, as well as to the intervention group (PRP+Physio, PPP + Physio, Physio) allocation. ${ }^{11}$ 


\section{Initial examination}

After initial examination by a sports medicine physician and MRI confirmation of a grade 1 or 2 hamstring strain injury ${ }^{12}$ (excluding grade III-complete rupture), the study participants presented to physiotherapy, where one of the three physiotherapists took their history and conducted a physical examination. Participants were questioned regarding the history of their injury, its mechanism and a range of subjective features, via use of a standardised form (see online supplementary material: initial and daily assessment forms and online supplementary video).

The examining (and treating) physiotherapist then performed a standardised physical examination, and documented palpation pain and location, strength, flexibility and functional testing results (see table 1 and online supplementary material: initial and daily assessment forms and online supplementary video).

After this initial assessment, an isokinetic evaluation of the uninjured leg was conducted to serve as a baseline measure. The injured athlete performed three isokinetic knee flexion and extension strength tests of the uninjured leg: at $60 \%$ s concentric extension and $60 \%$ s concentric flexion; $300 \%$ s concentric extension and $300 \%$ s concentric flexion; and $60 \%$ s eccentric extension and $180 \%$ s concentric flexion. ${ }^{13}$

The rehabilitation protocol has been previously described ${ }^{13}$ and is depicted in the online supplementary material.

\section{MRI}

MRI examination was performed prior to any injection being given. ${ }^{11}$ Patients were positioned supine and examined with a 1.5 Tesla Siemens Espree ${ }^{12}$ (Erlangen, Germany). In addition, a phased array coil and two body matrix coils were strapped over the thigh and centred over the painful area, as identified by the athlete. Axial and coronal proton density with fat saturation along the longitudinal axis of thigh (TR/TE 3490/27 and a $512 \times 326$ matrix for the coronal images and TR/TE 3000/32 and a $512 \times 333$ matrix for the axial images) with one signal average each were obtained.

Each MRI was assessed by one radiologist with more than 9 years of experience in musculoskeletal radiology. While the injured athletes had standardised MRI examinations performed at initial examination, and during their final discharge examination, only the initial examination MRI investigations are reported in this study. The radiologist was blinded to the clinical status and to information on whether the MRI was of the initial injury or at return to play.

For assessment of the MRIs, we used standardised scoring forms (see online supplementary material: MRI report sheet), which included the modification of Peetrons' grading; grade 0: no abnormalities; grade I: oedema without architectural distortion; grade II: oedema with architectural disruption; grade III: complete tear. ${ }^{12}{ }^{14}$ When more than one muscle was involved, the muscle with the most extensive oedema or disruption was scored. We measured the increased T2-signal intensity for the affected hamstring muscle in craniocaudal, transverse and anterioposterior dimensions on the fluid sensitive sequences (PD-FS). We recorded the distance from the ischial tuberosity, longitudinal length (craniocaudal) of the lesion and calculated the involved cross-sectional area as a percentage of the total muscle cross-sectional area in the transversal plane, and approximated the lesion volume. Excellent reliability, with the same radiologist involved, has been previously described. ${ }^{12}$

\section{Return to play}

The primary outcome was the time to return to play expressed as the number of days from injury (day 0 ) to the completion of the full rehabilitation programme and clearance to return to play by the treating sports medicine physician (blinded to the intervention). The treating sports medicine physician clinically reviewed athletes on a weekly basis and immediately on completion of the rehabilitation programme. The guidelines for making the final return to play decision included successful and asymptomatic completion of the progressive criteria-based rehabilitation programme, clinical evaluation and interpretation of the results of isokinetic assessment.

The return to play decision was step-wise, and involved, at first, completion of the 6 stage criteria-based rehabilitation programme, the final 3 of which are functional field-based stages specific to the individual athlete's normal practice regimen. On successful completion of these criteria, the athlete performed three isokinetic knee flexion and extension strength tests of both, the injured and the uninjured leg: at $60 \%$ soncentric extension and $/ 60 \%$ s concentric flexion; $300 \%$ s concentric extension and $300 \%$ s concentric flexion; and $60 \%$ s eccentric extension and $180 \%$ s concentric flexion. ${ }^{13}$ The same isokinetic protocol was performed on the uninjured leg prior to the start of the rehabilitation (baseline isokinetic). Isokinetic data guided the RTP decision, but there were no strict isokinetic criteria to be met. Informed by this, the treating sports medicine doctor then conducted a clinical examination and made a decision regarding return to play. ${ }^{13}$

\section{Statistical analysis}

The clinical utility of subjective and physical examination and the findings of acute MRI were investigated using linear regression. Statistical analysis was conducted using SPSS V.21 (IBM Corporation and other(s) 1989, 2012, Chicago, USA).

Three separate analyses were conducted to shed light on the utility of: (1) the initial physiotherapist's examination, (2) a combination of the initial and week one physiotherapist's examinations, and (3) the MRI examination alone and in combination with (1) and (2). For initial examination analysis, all the information collected at initial examination along with the MRI variables and the athlete's attendance at physiotherapy were made available for inclusion. For the week 1 analysis, all of these same

Table 2 Demographic data regarding the included injured athletes

\begin{tabular}{lc}
\hline & Values \\
\hline Age (years) & $25.8(5.8) 5.7)$ \\
Sports category & \\
Athletics & $4(4.4)$ \\
Basketball & $2(2.2)$ \\
Decathlon & $1(1.1)$ \\
Football & $66(73.3)$ \\
Futsal & $8(8.9)$ \\
Handball & $3(3.3)$ \\
Hockey & $2(2.2)$ \\
Physical Coach Football & $1(1.1)$ \\
Squash & $1(1.1)$ \\
Volleyball & $1(1.1)$ \\
Weightlifting and body building & $1(1.1)$ \\
Previous Hamstring injuries & $58(64.4)$ \\
Previous ipsilateral Hamstring injuries & $28(31.1)$ \\
\hline Age is presented as: mean (SD), sports category and past history of hamstring injury \\
are presented as numbers (percentage).
\end{tabular}


variables as well as the examination features from the day 7 examination were made available. For the MRI analysis, only the MRI variables were included.

To minimise the possibility of overfitting the data, in the first instance, simple scatter plots of each of the individual candidate predictor variables were created and correlations estimated, along with measures of central tendency (mean, median) depending on the kurtosis of the data, as well as estimates of variance (SD or IQR as appropriate) and estimates of covariance including variance inflation factor. Where this analysis suggested a relation, and the experienced clinicians involved in the study proposed a biologically plausible mechanism for their relation to return to play duration, the variable was retained for subsequent inclusion in the regression model, otherwise it was discarded. ${ }^{15} 16$ The suitable candidate variables were then examined in a multiple linear regression model with the independent variable being time taken (in days) from initial injury until medical clearance was given for return to full training and play.

Multiple linear regression was conducted using a best subsets approach, with the criteria for entry being improvement in adjusted $\mathrm{R}^{2}$ (variance explained adjusted for the number of predictors in the model). Equations for estimating RTP at initial examination, 1 week examination and using the MRI were generated along with estimations of the variance explained using these equations. Scatter plots were generated for the predicted and actual days of return to play along with the regression line and $95 \%$ CIs.

Table 3 Summary of final variables considered in the regression models

\begin{tabular}{|c|c|c|}
\hline Description & Abbreviation & Values \\
\hline Maximum pain (VAS) at the time of the injury reported by the athlete $(0-10)$ & $\operatorname{MaxP}$ & 6.9 (6.50 to 7.31$), 7.00(3.00-10.00)(85)$ \\
\hline $\begin{array}{l}\text { Strength of the injured (Inj) or uninjured (Uninj) leg in kg using a hand held dynamometer in the } \\
\text { 'Outer Range' position }\end{array}$ & $\begin{array}{l}\text { Outerlnj/ } \\
\text { OuterUninj }\end{array}$ & 14.4 (12.71 to 16.13$), 11.90(2.40-36.90)(80)$ \\
\hline $\begin{array}{l}\text { Strength of the injured (Inj) or uninjured (Uninj) leg in kg using a hand held dynamometer in the } \\
\text { 'Mid Range' position }\end{array}$ & Midlnj/MidUninj & 13.4 (11.50 to 15.27$), 11.20(2.00-35.20)(84)$ \\
\hline $\begin{array}{l}\text { Strength of the injured (Inj) or uninjured (Uninj) leg in kg using a hand held dynamometer in the } \\
\text { 'Outer Range' position }\end{array}$ & InnerInj/InnerUinj & $12.6(11.45$ to 13.78$), 12.20(3.00-26.00)(85)$ \\
\hline Athlete reports no pain during 'outer range' strength testing with HHD (yes/no) & PainlessOuterlnj & Yes $=58, \mathrm{No}=15$ \\
\hline Athlete reports no pain during 'mid range' strength testing with HHD (yes/no) & PainlessMidlnj & Yes $=51, \mathrm{No}=25$ \\
\hline Athlete reports no pain during 'inner range' strength testing with HHD (yes/no) & PainlessInnerlnj & $\mathrm{Yes}=43, \mathrm{No}=33$ \\
\hline $\begin{array}{l}\text { Strength of the injured leg when tested in the 'outer range' position expressed as a percentage of } \\
\text { the uninjured leg in the same position }\end{array}$ & Outer\% & $0.5(0.45$ to 0.57$), 0.43(0.08-1.13)$ \\
\hline $\begin{array}{l}\text { Strength of the injured leg when tested in the 'outer range' position expressed as a percentage of } \\
\text { the uninjured leg in the same position }\end{array}$ & Mid\% & $0.5(0.45$ to 0.57$), 0.43(0.09-1.21)$ \\
\hline $\begin{array}{l}\text { Strength of the injured leg when tested in the 'outer range' position expressed as a percentage of } \\
\text { the uninjured leg in the same position }\end{array}$ & Inner\% & $0.6(0.58$ to 0.69$), 0.63(0.12-1.47)$ \\
\hline Athlete plays football & SportFootball & $\mathrm{N}=64$ \\
\hline Athlete plays basketball & SportBasketball & $\mathrm{N}=4$ \\
\hline Athlete plays volleyball & SportVolleyball & $\mathrm{N}=1$ \\
\hline Athlete plays handball & SportHandball & $\mathrm{N}=3$ \\
\hline Track and field athlete & SportAthletics & $\mathrm{N}=4$ \\
\hline Athlete plays futsal & SportFutsal & $N=5$ \\
\hline Athlete plays a different sport & Sport0ther & $N=5$ \\
\hline $\begin{array}{l}\text { Hamstring flexibility test: passive knee extension with the hip in } 90^{\circ} \text { of flexion, supine; Injured/ } \\
\text { Uninjured leg }\left({ }^{\circ}\right)\end{array}$ & PKETInj/ PKETUninj & $\begin{array}{l}56.2(49.64 \text { to } 62.85), 55.00(-28.00 \text { to } 160.00) \\
83.5 \text { (80.09 to } 86.98), 90.00(40.00-125.00)\end{array}$ \\
\hline Hamstring Flexibility test: passive straight leg raise; Injured/Uninjured leg $\left({ }^{\circ}\right)$ & SLRInj/SLRUninj & $\begin{array}{l}64.3(59.07 \text { to } 69.49), 65.50(14.00-160.00) \\
89.5(85.98 \text { to } 93.01), 91.00(58.00-121.00)\end{array}$ \\
\hline $\begin{array}{l}\text { Player has had a previous hamstring injury on both legs (compared to no past history, or an injury } \\
\text { on one leg only) (yes/no) }\end{array}$ & PrevHSInjBoth & $\begin{array}{l}\mathrm{N}=31 \text { (no previous injury }=18 \text {, one leg only } \\
\text { previously injured }=34 \text { ) }\end{array}$ \\
\hline Per cent of appointments attended of available appointments (maximum 5 days per week) & Attendance $\%$ & $0.8(0.79$ to 0.87$), 0.89(0.00-1.00)$ \\
\hline Peak torque of knee flexion tested at tested at $60 \% \mathrm{~s}$, concentrically $(\mathrm{N} \cdot \mathrm{m})$ & PTH60c & $\begin{array}{l}112.3(107.37 \text { to } 117.16), 112.00(61.40- \\
156.10)\end{array}$ \\
\hline Angle of peak torque of the quadriceps, tested at $60^{\circ} / \mathrm{s}$, concentrically, uninjured leg $\left(^{\circ}\right)$ & APTQ60Uninj & $67.8(64.86$ to 70.80$), 70.00(14.00-89.00)$ \\
\hline $\begin{array}{l}\text { Change in strength of the injured leg, expressed as a percentage of the week } 1 \text { strength value of } \\
\text { that leg, tested in 'inner range' }\end{array}$ & Wk1 $\Delta$ Innerlnj & 10.8 (9.41 to 12.10$), 10.54$ (-4.33 to 24.98$)$ \\
\hline $\begin{array}{l}\text { Change in strength of the injured leg, expressed as a percentage of the week } 1 \text { strength value of } \\
\text { that leg, tested in 'mid range' }\end{array}$ & Wk1 $\triangle$ Midlnj & 11.1 (8.99 to 13.22$), 9.59$ (-3.62 to 34.01$)$ \\
\hline $\begin{array}{l}\text { Change in strength of the injured leg, expressed as a percentage of the week } 1 \text { strength value of } \\
\text { that leg, tested in 'outer range' }\end{array}$ & Wk1 $\triangle$ Outerlnj & 12.3 (10.38 to 14.25$), 9.69(-4.68$ to 35.70$)$ \\
\hline Days until player reports pain free walking (maximum 7) & TimeWalk & 2.9 (2.31 to 3.43$), 2.00(0.00-10.00)$ \\
\hline $\begin{array}{l}\text { Athlete is unable to painlessly perform } 3 \text { repetitions of a single leg bridge exercise painlessly on } \\
\text { the injured leg (yes/no) }\end{array}$ & SLBridgePain & $\mathrm{N}=29$ (44 athletes able to perform painlessly) \\
\hline Number of days delay from injury to starting physiotherapy & DelayRx & 3.3 (3.03 to 3.66$), 3.00(0.00-8.00)$ \\
\hline Volume of oedema within the injured muscle group as seen on MRI, measured in $\mathrm{cm}^{3}$ & VolOedema & 73.4 (53.93 to 92.86$), 31.87(0.00-409.98)$ \\
\hline
\end{tabular}

Values column represents mean $(95 \% \mathrm{Cl})$, median (minimum-maximum) $(\mathrm{N})$ except where the data are simple counts.

HHD, hand held dynamometry; MaxP, maximum pain at the time of injury; PTH, peak torque hamstrings; RTS: return to sports; SLR, straight leg raise flexibility; TR/TE, relaxation time/ echo time; VAS, visual analogue scale. 
Table 4 Patient history and physical examination variables retained in the regression equations

Initial examination
Injury and patient history
Maximum pain at the time of injury
Plays volleyball
Past history of hamstring injury in both legs
Athlete's attendance to physiotherapy (per cent of available appointments attended)
Physical examination
Passive knee extension range with the hip flexed to $90^{\circ}$
Straight leg raise range
Presence of pain during the 'outer range' strength test
'Mid range' strength expressed as a percentage of the uninjured leg
Angle of peak torque for knee extension at $60^{\circ} / \mathrm{s}$ (concentric)

Follow-up (day 7) examination

Maximum pain at the time of injury

Plays football

Number of days to walk pain free

Delay to starting physiotherapy

Change in strength for the 'Mid Range' test during the first 7 days Strength of the injured leg at initial examination in the 'Inner Range' test Presence of pain during the 'outer range' strength test

'Outer range' strength expressed as a percentage of the uninjured leg Pain when performing the Single leg bridge test (at day 7) Peak torque for knee flexion at $60 \%$ s (concentric)

\section{Note that while these can be considered the only useful variables of those examined in relation to predicting time to return to play, it is suggested that only the combination of} variables depicted in the follow-up examination are clinically useful given the width of the $95 \% \mathrm{Cls}$.

\section{RESULT}

From November 2009 through October 2013, 90 patients were included. The baseline demographic variables are presented in table 2 .

The mean duration between injury and initial assessment was 2.0 days (SD 1.1). The mean duration of return to play was 25.1 days (10.1). There were 46 grade I and 44 grade 2 injuries with a mean oedema volume of $70.9(89.2) \mathrm{cm}^{3}$.
The variables retained for the predictive analysis are shown in table 3 .

At initial examination, a combination of nine variables (6 clinical and 3 demographic) in a regression equation explained 59\% of the variance in the time to return to play. The six clinical variables that predicted return to play were:

Maximum pain at the time of injury (MaxP), presence of pain on the 'outer range' strength test, the mid-range strength test

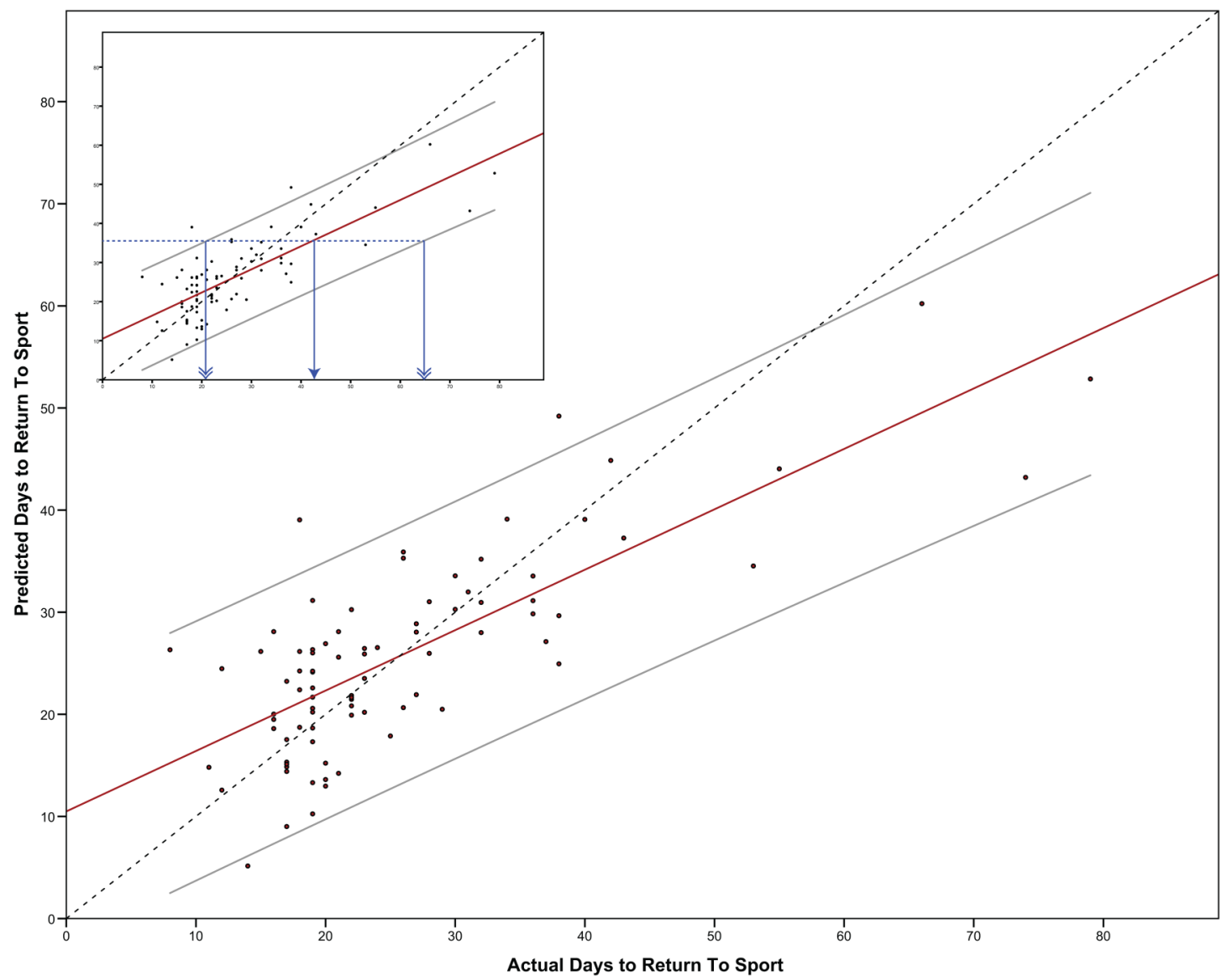

Figure 1 Scatter plot of the regression equation for initial physical examination findings (Days to Return to Sport Predicted) against actual time to return to sport (Days to Return to Sport Actual) with $95 \% \mathrm{Cl}, \mathrm{R}^{2}=0.592, \mathrm{p}<0.01$. Dashed line represents perfect agreement reference line, that is, where the predicted value is equal to the actual value. Inset: How to interpret the scatter plot if the equation predicted 35 days return to play. The horizontal (dotted) line intersects the regression line at 42 days actual return to play (single arrowhead) and the $95 \% \mathrm{Cl}$ ranges from 21 days to 65 days (double arrowheads). Thus, this set of variables is not helpful for a player or coach. 
expressed as a percentage of the uninjured leg, passive knee extension range in $90^{\circ}$ hip flexion ('PKET'), Straight Leg Raise flexibility and the angle of peak torque for knee extension at $60 \%$ s (concentric).

The three demographic variables that predicted return to play were:

Playing the sport of volleyball, a history of hamstring injury on both legs and the athlete's attendance to physiotherapy (expressed as a percentage of possible attendance). These variables are outlined in table 4 .

Variables associated with a longer time to return to play will have a positive coefficient, while variables associated with a shorter return to play will have a negative coefficient. The coefficient multiplied by the variable (in its units of measurement) are then the associated change in expected return to play time. For example, MaxP has a coefficient of +2.4 . This can be interpreted as: if an athlete reported $8 / 10$ as their MaxP, then this would be associated with 19.2 days (ie, $2.4 \times 8$ ) longer return to play duration. Variables that are 'Yes/No' are interpreted as 1 or 0 , respectively. For example, if, on initial examination, the athlete reported pain when performing the outer range strength test ('OuterInjP'), this was associated with a shorter return to play duration of 11.25 days (ie, $11.25 \times 1$ ).

At initial examination, variables associated with a longer time to return to play were: higher MaxP, and greater passive flexibility of knee extension of the uninjured leg with the hip flexed to $90^{\circ}$ (PKETUninj) and a higher angle of peak torque for knee extension in the uninjured leg (Quadriceps) tested at $60 \%$ (APTQ60Uninj). Variables associated with a shorter duration of return to play were: presence of pain on outer range strength testing (OutInjP), greater strength of the injured leg as a percentage of the uninjured leg when tested in the 'mid range' position expressed as a percentage (Mid\%), playing the sport of volleyball (SportVolleyball), greater passive straight leg raise flexibility of the uninjured leg (SLRUninj), history of a hamstring injury to both legs (PrevHSInjBoth) and higher number of days attending physiotherapy expressed as a percentage of possible days attendance (attendance\%) (see table 3, equation 1).

The 95\% CI surrounding this equation at the median return to play time covers approximately 46 days, that is, \pm approximately 23 days (see equation 1 , and figure 1 ).

$$
\begin{aligned}
\text { Days } 1= & -4.3+2.4(\operatorname{maxP})-11.25(\text { OutInjP })-16.5(\mathrm{Mid} \%) \\
& -8.1(\text { SportVolleyball })+0.37(\text { PKETUninj }) \\
& -0.33(\text { SLRUninj })-5.4(\text { PrevHSInjBoth }) \\
& -15.5(\text { Attendance } \%)+0.24(\text { APTQ60Uninj })
\end{aligned}
$$

At 1 week post initial examination, a combination of 10 (9 physical and 1 demographic) variables explained $97.0 \%$ of the variance in time to return to play. Variables associated with a

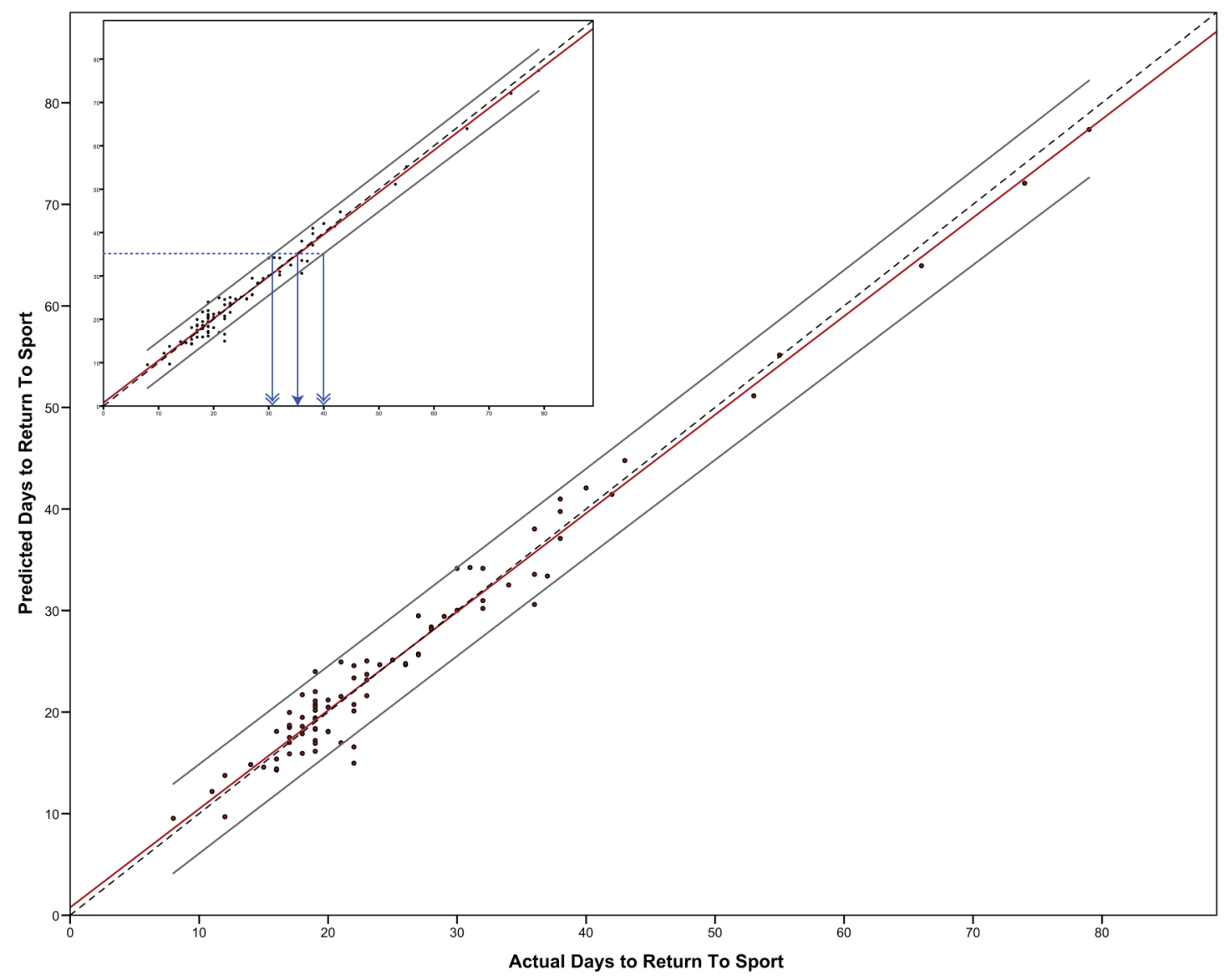

Figure 2 Scatter plot of the regression equation for initial examination and week 1 postinjury examination findings (Days to Return to Sport Predicted) against actual time to return to sport (Days to Return to Sport Actual) with $95 \% \mathrm{Cl}, \mathrm{R}^{2}=0.970, \mathrm{p}<0.01$. Dashed line represents perfect agreement reference line, that is, where the predicted value is equal to the actual value. Inset: How to interpret the scatter plot if the equation (see online spreadsheet calculator) predicted 35 days return to play. The horizontal (dotted) line intersects the regression line at 35 days actual return to play (single arrowhead) and the $95 \% \mathrm{Cl}$ ranges from 31 days to 40 days (double arrowheads). Thus, this set of variables is likely helpful for a player or coach. 
shorter return to play duration were: greater improvement in strength of the 'mid-range' strength test over the 7 day period (Wk1 $1 \Delta$ MidInj), a painless strength test in the 'Outer range' position and playing the sport of Football (SportFootball).

Variables associated with a longer duration of return to play were: greater isokinetic strength of the knee flexors when tested at $60 \%$ on the uninjured leg (PTHC60U), higher maximum pain reported at the time of injury (MaxP), higher number of days taken to walk pain free (TimeWalk), greater strength on the 'Inner Range' strength test on the injured leg (InnerInj), pain reported when attempting a single leg bridge exercise (SLBridgePain), greater delay in starting physiotherapy after injury and higher percentage of strength tested in the 'Outer Range' position (injured compared to uninjured, outer\%). The 95\% CI surrounding this equation covers approximately 10 days, that is, $4 \pm$ approximately 5 days (see equation 2 , and figure 2 ).

$$
\begin{aligned}
\text { Days7 }= & -212.8-2.6(\mathrm{Wk} 1 \Delta \mathrm{idInj})+0.4(\mathrm{PTHC} 0 \mathrm{U}) \\
& +405(\mathrm{MaxP})+3(\text { TimeWalk })-13(\text { SportFootball }) \\
& +13.3(\text { InnerInj })+16.3(\text { SLBridgePain }) \\
& -12.8(\text { PainlessOuterInj })+3.3(\text { DelayRx }) \\
& +0.56(\text { Outer } \%)
\end{aligned}
$$

Examination of the MRI variables only and their relation to return to play duration showed a significant relation only for the volume of oedema (in $\mathrm{cm}^{3}$ ) such that the number of days predicted to return to play was equal to this volume $\times 0.11$ +19.7 days $(\mathrm{p}<0.05)$, however, the variance explained was only $8.6 \%$, which resulted in a $95 \% \mathrm{CI}$ in excess of 80 days for the median time to return to play (see figure 3 and equation 3). A box plot of days taken to return to play compared with overall MRI grading is shown in figure 4. Note that while all MRI variables were available for inclusion in the first two models, none were ultimately retained.

$$
\text { DaysMR }=-19.7+0.11(\text { VolOedema })
$$

\section{DISCUSSION}

These data show that the combination of initial and follow-up (7 days) examination has clinical utility, explaining 97\% of the total variance of time to return to play in acute hamstring injuries. However, a single clinical examination at initial presentation is clinically less helpful in determining time to return to play (explaining 59\% of the total variance).

\section{Predicting time to return to play from these data- information for coaches and players}

To understand the utility of the described regression equations, the predicted and actual return to play values, along with their 95\% CI's, need to be examined. These values can be estimated

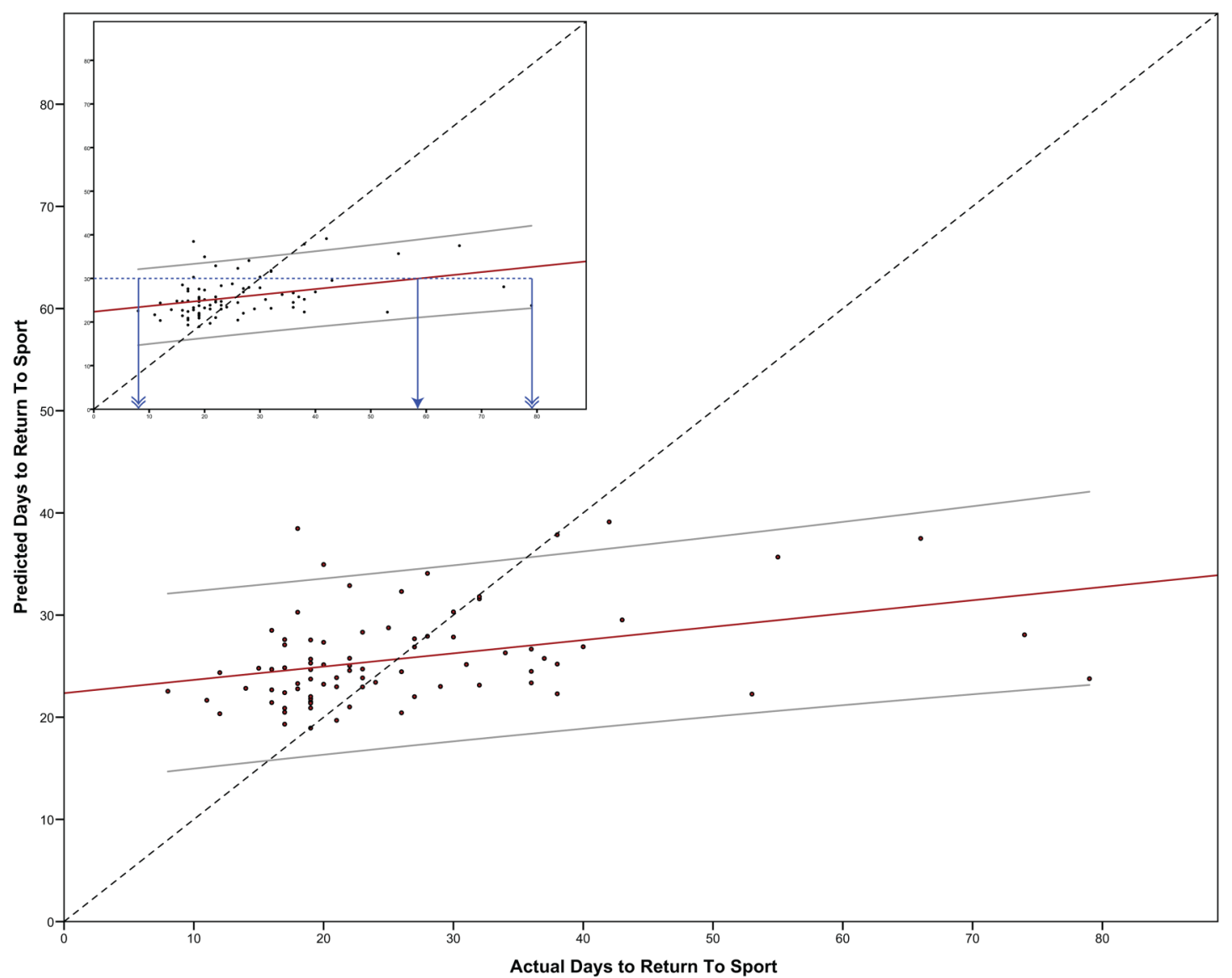

Figure 3 Scatter plot of the regression equation for MRI findings (Days to Return to Sport Predicted) against actual time to return to sport (Days to Return to Sport Actual) with $95 \% \mathrm{Cl}, \mathrm{R}^{2}=0.130, \mathrm{p}<0.05$. Dashed line represents perfect agreement reference line, that is, where the predicted value is equal to the actual value. Inset: How to interpret the scatter plot if the equation predicted 40 days return to play. The horizontal (dotted) line intersects the regression line at 58 days actual return to play (single arrowhead) and the $95 \% \mathrm{Cl}$ spans the range of the entire data set, that is, 7-78 days (double arrowheads). As such, MRI is entirely unhelpful in predicting return to play duration. 


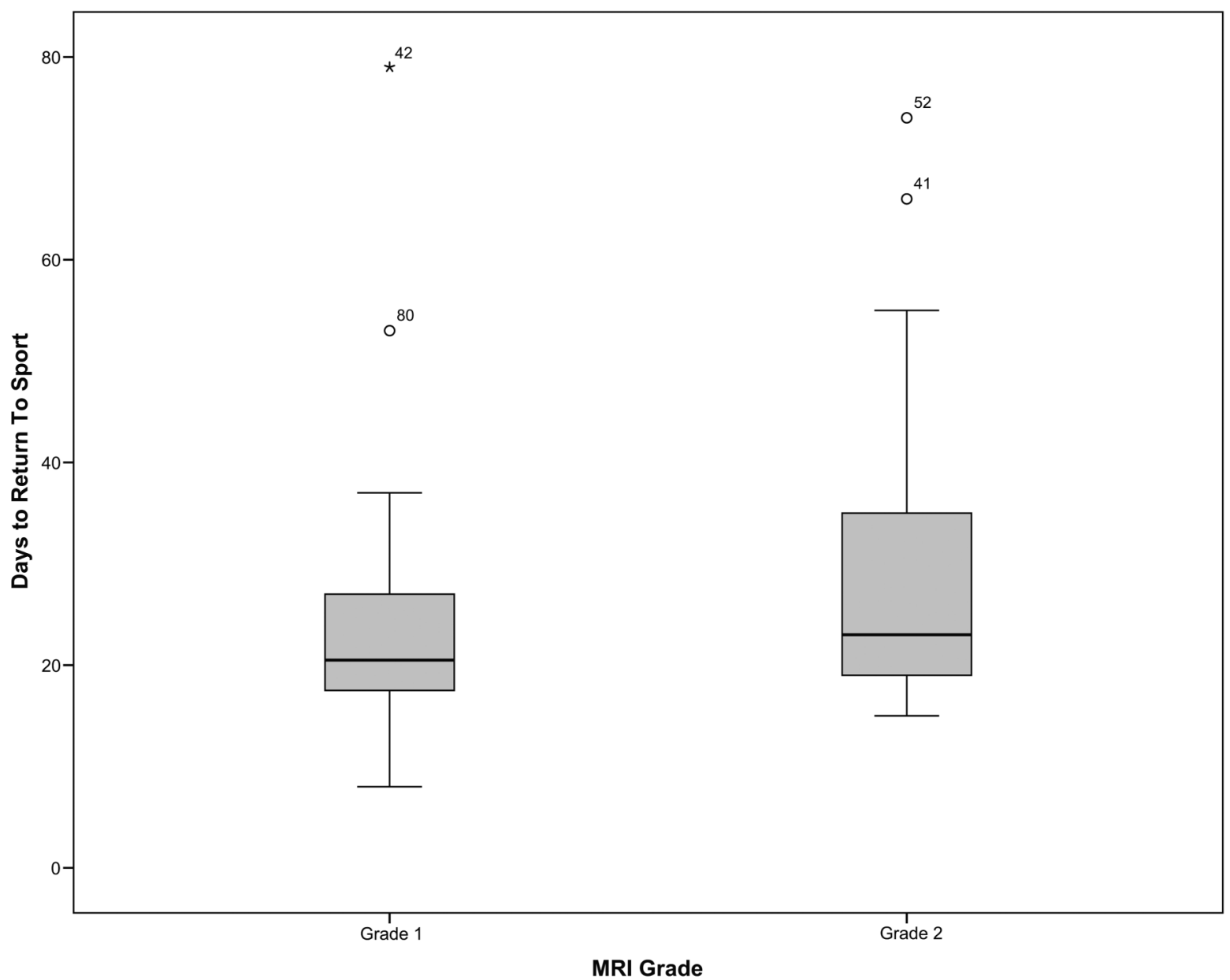

Figure 4 Days taken to return to sport compared with MRI grading. No significant difference between the grades 1 (median RTP: 20.5, IQR: 9.8) and 2 (Median: 23, IQR: 17.5) was found, Mann-Whitney U: two-tailed asymptotic significance: 0.618 . Note that 4 outliers are depicted in this analysis. Open circles (Case 80 in grade 1, and cases 41 and 52 with a grade 2 injury) are participants who were $>1.5$ times the IQR, and an asterisk denotes participant 42 , who was $>3$ times the IQR.

from the equations and the figures provided. A predicted return to play value can be calculated from the provided formulae, and the $95 \%$ CI associated with a predicted value can be estimated graphically from the figures provided allowing the reader to derive clinical importance. To do this, choose a predicted value of interest (on the vertical-axis, or Y-axis) and then trace a line across horizontally until it intersects with the 95\% CI values on the plot. The range of the horizontal (X-axis) values included between these intersections is the $95 \% \mathrm{CI}$, in days.

For example, taking the mean return to play duration (approximately 25 days) and using figure 1, reflecting initial examination variables, the actual value for return to play is approximately 24 days, however, the $95 \%$ CI associated with this prediction is from 8 to 47 days. Using figure 3 (initial and follow-up examination), the actual return to play value is approximately 25 days, with a 95\% CI from 21 to 29 days. Using figure 4, the MRI examination, the actual return to play is 27 days, however, the $95 \%$ CI spans the entire range of return to play values-from 8 to 78 days.

We suggest that, from these data, clinically meaningful inferences can only be made from the data taken at initial examination and 7 days postinjury, despite the statistical significance of this equation. Conversely, clinically meaningful information was available from neither the initial examination nor from the MRI examination (nor any combination of variables taken at this time).

\section{MRI did not add value in predicting return to play-why not?}

Interestingly, the MRI features were not retained in either of the analyses that included clinical features, suggesting that MRI offered no added value in predicting return to play duration. Similarly, injection of PRP or PPP, or no injection, was not found to significantly alter the return to play duration in this cohort. ${ }^{11}$ The combination of initial and follow-up examination has not been studied before, but clearly reflects daily practice while dealing with acute injuries. Previous studies included only single baseline assessments and reported even lower percentages of explained total variance. ${ }^{17}$

In contrast to other research showing an association between MRI parameters and time taken to return to play, ${ }^{18}$ we found no such utility. We suggest three possible reasons for this discrepancy. First, previous studies were based on univariate analysis with a high risk of bias. ${ }^{17}$ Second, this study only examined grades 1 and 2 hamstring injuries. We suspect that grades 0 and 3 will, respectively, show much shorter and longer time to return to play than grades 1 and 2. As such, correlation or regression analyses will have much greater proportion of variance explained by including these more extreme values. Finally, in our study, the treating therapists and medical practitioners were blinded to the MRI, and clinical decisions were made according to subjective reports of the patient and objective clinical (physical) findings. To our knowledge, only one previous 
paper examining return to play duration has blinded the clinical decision makers to the MRI. ${ }^{19}$ In that study, there was no correlation between MRI findings and time to return to play, either.

\section{Limitations}

While we have taken steps to minimise the possibility of the influence of overfitting, a significant limitation of this analysis is that the data are certainly overfit with a much higher number of candidate variables than should be permitted for an exploratory analysis. ${ }^{20}$ Accordingly, at least some of the variables will have been incorrectly retained by capitalising on chance. ${ }^{21}$ These analyses should be seen as preliminary, and will need to be confirmed in a subsequent study. ${ }^{22}$

A further limitation of this study is that those in the cohort examined were predominantly professional sportsmen (mainly football players) who had enrolled in an RCT where they could have been assigned an injection therapy. Most likely, such a cohort will vary from a recreational athlete, and likely even some other professional athletes who feel differently about being included in a randomised study for their injury.

Of the identified variables, time to walk pain free has previously been proposed, however, this was in a different population (AFL players), in which rehabilitation was not controlled. ${ }^{23}$

A corollary of the likelihood of false positive assessment findings is that those not showing an association with return to play in this analysis are unlikely to be clinical predictors. ${ }^{16}$

Of interest, the football players in this cohort displayed a shorter time to discharge than participants from the other sports, such as track and field athletes. We speculate that the differing physical demands of individual sports (and therefore the differing requirements to reach sports-specific goals during rehabilitation) are contributing factors for this result.

We believe that a significant strength of this study is the tightly controlled and standardised criteria-based rehabilitation. We feel that such an approach has minimised the variability of outcome associated with interindividual rehabilitation variation.

This study has not examined predictors of reinjury. Given the reported high recurrence of hamstring injury, the clinical and MRI factors examined here should be examined to see if they can help predict those athletes likely to be reinjured.

\section{Summary}

A combination of subjective and objective examination features gleaned from the initial physiotherapy examination, and a further examination 7 days later, predicted time to return to play in this large series of athletes with grade I and II hamstring strain injuries. The key variables that clinicians may wish to consider when examining similar patients are: the change in strength from initial examination to day 7 examination for the 'mid range' test; isokinetic knee extension strength of the uninjured leg on day 1; the maximum pain reported by the athlete at the time of injury; playing the sport of football; strength at initial assessment on the inner 'range' test for the injured leg; the presence of pain on performing a single leg bridge at day 7; the delay in starting treatment; and the strength of the injured

\section{What are the findings?}

Predicting return to play duration after hamstring injury at initial examination is difficult, and time to return to play varies widely.

\section{How might it impact on clinical practice in the future?}

A range of clinical examination findings performed at day 1 and day 7 of physiotherapy treatment likely offers reasonable predictive ability in duration of return to play. The key subjective features associated with return to play time were:

- Maximum pain reported at the time of injury, delay in starting physiotherapy,

- Time taken to walk pain free,

- Playing the sport of football.

The key physical findings were six strength testing related variables:

- Change in pain on the mid range strength test over the first week,

- Pain during the outer range strength test and single leg bridge at day 7 ,

- Outer range strength at day 7 expressed as a percentage of the uninjured leg,

- Strength during the inner range test at day 1 ,

- Peak isokinetic strength of knee flexion of the uninjured leg. MRI offered no additional clinically meaningful information.

leg (expressed as a percentage of the uninjured leg) in the 'outer range' test performed at day 1 . Conversely, examination performed on day 1 alone, as well as the addition of MRI examination, offered no clinical utility.

Contributors PJ, PM and RW conceived the study and collected the data. All the authors were involved in the analysis of the data. PJ, EW, RW and JT were responsible for writing the final manuscript.

Competing interests None declared.

Ethics approval Aspetar Sports Medicine Hospital Ethics Committee.

Provenance and peer review Not commissioned; externally peer reviewed.

\section{REFERENCES}

1 Malliaropoulos N, Papalexandris S, Papalada A, et al. The role of stretching in rehabilitation of hamstring injuries: 80 athletes follow-up. Med Sci Sports Exerc 2004:36:756-9.

2 Askling CM, Tengvar M, Saartok $T$, et al. Acute first-time hamstring strains during high-speed running: a longitudinal study including clinical and magnetic resonance imaging findings. Am J Sports Med 2007;35:197-206.

3 Askling CM, Tengvar M, Saartok T, et al. Proximal hamstring strains of stretching type in different sports: injury situations, clinical and magnetic resonance imaging characteristics, and return to sport. Am J Sports Med 2008;36:1799-804.

4 Askling CM, Malliaropoulos N, Karlsson J. High-speed running type or stretching-type of hamstring injuries makes a difference to treatment and prognosis. Br J Sports Med 2012:46:86-7.

5 Comin J, Malliaras P, Baquie P, et al. Return to competitive play after hamstring injuries involving disruption of the central tendon. Am J Sports Med 2013;41:111-15.

6 Malliaropoulos N, Isinkaye T, Tsitas $\mathrm{K}$, et al. Reinjury after acute posterior thigh muscle injuries in elite track and field athletes. Am J Sports Med 2011;39:304-10.

7 Askling CM, Tengvar M, Saartok T, et al. Acute first-time hamstring strains during slow-speed stretching: clinical, magnetic resonance imaging, and recovery characteristics. Am J Sports Med 2007:35:1716-24.

8 Sherry MA, Best TM. A comparison of 2 rehabilitation programs in the treatment of acute hamstring strains. J Orthop Sports Phys Ther 2004;34:116-25.

9 Askling CM, Tengvar M, Thorstensson A. Acute hamstring injuries in Swedish elite football: a prospective randomised controlled clinical trial comparing two rehabilitation protocols. Br J Sports Med 2013:47:953-9.

10 Askling CM, Tengvar M, Tarassova O, et al. Acute hamstring injuries in Swedish elite sprinters and jumpers: a prospective randomised controlled clinical trial comparing two rehabilitation protocols. Br J Sports Med 2014;48:532-9.

11 Hamilton $B, T o l J L$, Almusa $E$, et al. Platelet-rich plasma does not enhance return to play in hamstring injuries: a randomised controlled trial. Br J Sports Med 2015;49:943-50. 


\section{Original article}

12 Hamilton B, Whiteley R, Almusa E, et al. Excellent reliability for MRI grading and prognostic parameters in acute hamstring injuries. Br I Sports Med 2014;48:1385-7.

13 Tol JL, Hamilton B, Eirale C, et al. At return to play following hamstring injury the majority of professional football players have residual isokinetic deficits. Br I Sports Med 2014:48:1364-9.

14 Peetrons P. Ultrasound of muscles. Eur Radiol 2002;12:35-43.

15 Royston $P$, Moons KG, Altman DG, et al. Prognosis and prognostic research: developing a prognostic model. BMJ 2009;338:b604.

16 Moons KG, Royston $P$, Vergouwe $Y$, et al. Prognosis and prognostic research: what, why, and how? BMJ 2009;338:b375.

17 Reurink G, Brilman EG, de Vos RJ, et al. Magnetic resonance imaging in acute hamstring injury: can we provide a return to play prognosis? Sports Med 2015;45:133-46.
18 Gibbs NJ, Cross TM, Cameron M, et al. The accuracy of MRI in predicting recovery and recurrence of acute grade one hamstring muscle strains within the same season in Australian Rules football players. J Sci Med Sport 2004;7:248-58.

19 Moen MH, Reurink G, Weir $A$, et al. Predicting return to play after hamstring injuries. Br J Sports Med 2014;48:1358-63.

20 Tabachnick BG, Fidell LS. Using multivariate statistics. 5th edn. Boston: Pearson/ Allyn \& Bacon, 2007.

21 Vehkalahti K, Puntanen S, Tarkkonen L. Effects of measurement errors in predictor selection of linear regression model. Comput Stat Data Anal 2007;52:1183-95.

22 Altman DG, Vergouwe $Y$, Royston $P$, et al. Prognosis and prognostic research: validating a prognostic model. BMJ 2009;338:b605.

23 Warren $\mathrm{P}$, Gabbe BJ, Schneider-Kolsky M, et al. Clinical predictors of time to return to competition and of recurrence following hamstring strain in elite Australian footballers. Br J Sports Med 2010;44:415-19. 